\title{
Analysis of an Outbreak of Salmonella Enteritidis by Repetitive-Sequence-Based PCR and Pulsed-Field Gel Electrophoresis
}

\author{
Abdullah Kilic ${ }^{1}$, Orhan Bedir ${ }^{1}$, Nafiz Kocak ${ }^{2}$, Belkis Levent ${ }^{3}$, Can Polat Eyigun ${ }^{4}$, \\ Omer Faruk Tekbas ${ }^{5}$, Levent Gorenek ${ }^{4}$, Orhan Baylan ${ }^{1}$ and A. Celal Basustaoglu ${ }^{1}$
}

\begin{abstract}
Objective The aim of this study was to investigate a large food-borne outbreak associated with eggs contaminated by Salmonella Enteritidis in a military unit using pulse field gel electrophoresis (PFGE) and the Repetitive-sequence-based PCR (rep-PCR) employing the DiversiLab system.

Materials and Methods In mid-January 2008, a food-borne outbreak associated with $S$. Enteritidis occurred in a military unit located in the city centre of Isparta. A total of 2,469 patients were registered to six hospitals with gastrointestinal disease symptoms such as diarrhea and abdominal pain. Of those registered, 445 were hospitalized. $S$. Enteritidis was isolated from 276 stool samples and a blood sample of the hospitalized patients and from a food item. The PFGE patterns after XbaI digestion and rep-PCR profiles produced by the DiversiLab system ${ }^{\mathrm{TM}}$ were determined for eight randomly selected stool isolates, one blood isolate and one food isolate.

Results The PFGE patterns of all isolates were identical. The Rep-PCR profiles produced by using the DiversiLab system showed that all isolates were indistinguishable. The PFGE and rep-PCR interpretations were concordant for the $S$. Enteritidis isolates. All stool isolates, one blood isolate and one food isolate were susceptible to all antibiotics tested.

Conclusion This data suggest that the DiversiLab system may be a reasonable alternative to PFGE for investigation and control of $S$. Enteritidis outbreaks, since it is easy to use, rapid and does not require highly skilled operators.
\end{abstract}

Key words: $S$. Enteritidis, outbreak, PFGE, rep-PCR, DiversiLab system

(Inter Med 49: 31-36, 2010)

(DOI: 10.2169/internalmedicine.49.2743)

\section{Introduction}

Salmonella bacteria are one of the most important pathogens causing human illness. Foodborne diseases have been estimated to cause 200,000 deaths each year (1). More than 2,500 serovars of Salmonella enterica have been reported from all over the world. Salmonella enterica subsp. enterica serovar Enteritidis ( $S$. Enteritidis) is the most common se- rovar of Salmonella isolated from humans, animals, food and feed in the past 10 years, particularly in many developed countries (2).

$S$. Enteritidis caused 29,762 illnesses, 2,904 hospitalizations, and 79 deaths in the United States from 1985 to 1999 (3). In Europe, S. Enteritidis emerged as the most often reported serovar since the middle of the 1980s (4). In ten Turkish provinces, $S$. Enteritidis was also found as the most prevalent serovar isolated from various clinical sam-

${ }^{1}$ Department of Microbiology and Clinical Microbiology, Gulhane Military Medical Academy and School of Medicine, Ankara, Turkey, ${ }^{2}$ Isparta Military Hospital, Isparta, Turkey, ${ }^{3}$ Communicable Diseases Research Department, Refik Saydam National Hygiene Center, Ankara, Turkey, ${ }^{4}$ Department of Infectious Diseases, Gulhane Military Medical Academy and School of Medicine, Ankara, Turkey and ${ }^{5}$ Department of Public Health, Gulhane Military Medical Academy and School of Medicine, Ankara, Turkey

Received for publication August 5, 2009; Accepted for publication September 2, 2009

Correspondence to Dr. Abdullah Kilic, abkilic@gata.edu.tr 
ples (5).

$S$. Enteritidis is also one of the major food-borne pathogens associated with outbreaks (6). S. Enteritidis infections in an outbreak are usually acquired by ingestion of contaminated food items, such as eggs, milk, vegetables and meat or through animal contact $(7,8)$. Numerous phenotypic and genotyping methods have been used to identify food-borne disease outbreak caused by $S$. Enteritidis, including phage typing, serotyping, antibiogram, pulsed-field gel electrophoresis (PFGE), ribotyping, random amplified polymorphic DNA, and multisequence locus typing, etc. (2). The PFGE has been proven to be useful for the discrimination and epidemiological characterization of $S$. Enteritidis strains and is currently known to be the gold standard. Due to the high genetic homogeneity of $S$. Enteritidis, the combination of several methods has been suggested for the epidemiological analysis of unrelated and related $S$. Enteritidis outbreak strains $(2,9,10)$.

PCR-based methods are rapid, simpler, easy to perform and less costly than PFGE (11). Repetitive-sequence-based PCR method (rep-PCR) has been used to subtype a variety of bacteria with much success for over 10 years (12). This method uses primers that target non-coding repetitive sequences interspersed throughout the bacterial and fungal genome. A semi-automated rep-PCR assay system was commercially changed to the DiversiLab system which allows simplistic data elaboration, archiving fingerprint patterns, and reporting $(13,14)$.

The aim of this study was to investigate a large foodborne outbreak associated with eggs contaminated by $S$. Enteritidis in a military unit using PFGE and rep-PCR employing the DiversiLab system.

\section{Materials and Methods}

\section{Definition of the outbreak}

A large food-borne outbreak occurred in a military unit located in the city centre of Isparta. During the time of outbreak 6,418 soldiers were staying in this military unit. There were two kitchens to cook meals. The food-borne outbreak was based in one of the kitchens. According to the history of food consumption, all ill soldiers ate meatballs, lentil soup, mashed potatoes with egg, leek, and small cakes with syrup at 19:00 dinner time on Friday, January 12, 2008. A case was defined as having had meals in the military unit, and had developed diarrhea in addition to at least one of the gastrointestinal symptoms including fever $\left(>38.5^{\circ} \mathrm{C}\right)$, vomiting, nausea, headache or abdominal pain.

\section{Microbiological analysis}

Stool samples were collected from 445 hospitalized soldiers and using processed standard methods with some modification in six different hospitals. Briefly, stool samples were inoculated on Selenit F broth (Merck, Darmstadt, Germany). After 6-8 hours of incubation, the samples were im- mediately subcultured onto Salmonella-Shigella agar (Merck) and MacConkey agar (Merck) plates. All plates were incubated for up to 3 days at 35 to $37^{\circ} \mathrm{C}$. After incubation, colonies were identified on the basis of biochemical tests and confirmed as Salmonella spp. by the BD Phoenix Automated Microbiology System (Becton Dickinson Diagnostic Systems, Sparks, MD, USA) (7, 15). Serotyping of $S$. Enteritidis strains was determined according to the Kauffmann-White method by using agglutination with specific antisera (Statens Serum Institut, Copenhagen, Denmark) according to the manufacturer's instructions (16). Antibiotic susceptibility testing for $S$. Enteritidis strains was performed by using the BD Phoenix Automated Microbiology System (Becton Dickinson Diagnostic Systems). When suspected bacteremia, patients' blood samples were processed from two different venous vessels with the Bactec 9240 non-radiometric blood culture system (Becton Dickinson, Detroit, MI, USA). If the two blood cultures become positive, they were sub-cultured onto MacConkey (Merck) and 5\% sheep blood agar plates (Merck). Various food and environmental samples (kitchen items, sinks, and taps) were also collected for laboratory analysis to identify the causal agents and then cultured with methods as described above $(7,15)$.

\section{Pulse field gel electrophoresis method}

$S$. Enteritidis cells were grown overnight on brain heart infusion broth (Merck). Bacterial cells suspension consisting of approximately $2 \times 10^{9}$ cells $/ \mathrm{mL}$ were mixed with an equal volume of $2 \%$ low-melting-point agarose at $50^{\circ} \mathrm{C}$. The cellagarose suspension was pipetted into a block mold and was allowed to solidify at $4^{\circ} \mathrm{C}$. The cells were lysed at $50^{\circ} \mathrm{C}$ overnight with gentle shaking in a lysis buffer $(100 \mu \mathrm{g}$ of proteinase $\mathrm{K}$ per $\mathrm{mL}$ and $1 \%$ lauroylsarcosine in $0.5 \mathrm{M}$ EDTA). The blocks were washed three times in TE buffer (10 mM Trizma base, $1 \mathrm{mM}$ EDTA) at $4^{\circ} \mathrm{C}$ for 10 minutes and were stored at $4{ }^{\circ} \mathrm{C}$. An agarose block was equilibrated in restriction enzyme buffer for 30 minutes first and then digested with $40 \mathrm{U}$ of $\mathrm{Xba \textrm {I }}$ for 4 hours according to the manufacturer's instructions. The restricted blocks were washed in TE buffer for 60 minutes at $37^{\circ} \mathrm{C}$ and then stored at $4{ }^{\circ} \mathrm{C}$. The blocks were loaded into the wells of a $1 \%$ agarose gel. Electrophoresis was performed in $0.53 \mathrm{TBE}$ buffer (44.5 mM Trizma base, $44.5 \mathrm{mM}$ boric acid, $1 \mathrm{mM}$ EDTA) by the contour-clamped homogeneous electric field method with a CHEF-DRII drive module (Bio-Rad Laboratories Ltd., Hemel Hempstead, United Kingdom). To achieve optimal separation of the fragments, XbaI-digested DNA was electrophoresed with a run time of 18 hours under 2.2 to 63.8 seconds linear ramped pulse times. The gels were stained with ethidium bromide $(1 \mu \mathrm{g} / \mathrm{mL})$ for 40 to $60 \mathrm{~min}$ utes (17). Banding patterns were photographed under UV transillumination and analyzed with the aid of Quantity One software (Biorad/USA). Loci were scored for the presence or absence of a band. For dendrogram construction, a $2 \%$ difference in band position was tolerated. The percent simi- 
larity of the banding patterns was estimated with the Dice coefficient (18).

\section{Rep-PCR using DiversiLab system}

$S$. Enteritidis DNA was extracted as described Kilic et al (19). Rep-PCR was performed by using the DiversiLab Salmonella Kit for DNA fingerprinting (bioMerieux, Marcyl'Etoile, France) according to the manufacturer's instructions. Briefly, $50 \mathrm{ng}$ of genomic DNA, $2.5 \mathrm{U}$ of AmpliTaq, and 1.5 of 10X PCR buffer were added for a total of $25 \mu \mathrm{L}$ per reaction mixture. Thermal cycling parameters were as follows: initial denaturation of $94^{\circ} \mathrm{C}$ for $30 \mathrm{~s}$, annealing at $50^{\circ} \mathrm{C}$ for $30 \mathrm{~s}$, extension at $70^{\circ} \mathrm{C}$ for $90 \mathrm{~s}$; and a final extension at $70^{\circ} \mathrm{C}$ for 3 minutes. Analysis of PCR products were implemented by using DiversiLab System (bioMerieux) in which the amplified fragment of various size and fluorescent intensities were separated and detected using a microfluidics chip with the Aligent 2100 Bioanalyzer (Aligent Technologies, Santa Clara, CA, USA). Further analysis was performed with the web-based DiversiLab software version 3.3 with the Pearson correlation coefficient to determine distance matrices and the un-weighted-pair group method with arithmetic mean to create dendrograms. The resulting DNA fingerprinting patterns were viewed as electropherograms, and the report included a dendrogram constructed from a similarity matrix and a virtual gel image of the fingerprint for each DNA sample. Isolates were categorized as "different", "similar" and "indistinguishable" by cluster analysis and guidelines provided by the manufacturer. In general, "different", "similar" and "indistinguishable" were defined as $<95 \%,<97 \%$ and $>95 \%$ similarity, respectively (20).

\section{Results}

\section{Definition of the outbreak}

All patients were male. Their ages ranged from 21 to 29 old (median 23 ages). The 2,469 soldiers (38.4\% of the all 6,418 person) having gastroenteritis had consumed the same kind of meal of mashed potatoes with egg. Among these soldiers, $445(18 \%)$ were admitted to six different hospitals in Isparta during the outbreak period. The first case was hospitalized on Friday January 12, 2008 within 5 hours of having suspicious meals and then the others continued to visit a hospital in subsequent hours. Incubation period among those who ate the suspected meals varied between 5 and 96 hours. The average length of incubation was 46 hours. The main symptoms reported were diarrhea (100\%), abdominal pain (93\%), fever $(91 \%)$, nausea $(82 \%)$, headache (78\%), and vomiting (74\%). They were given ciprofloxacin (500 mg bid for 7 days), buscopan and total parenteral nutrition solution. Mortality did not occur among these patients during hospitalization. However, four patients required kidney dialysis due to acute renal failure after dehydration.

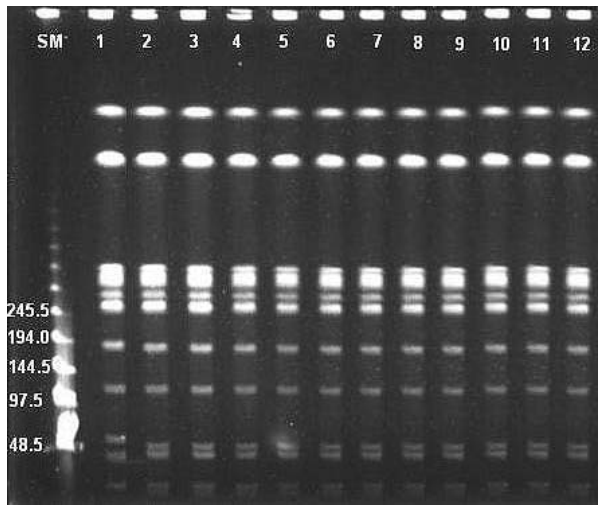

Figure 1. Pulsed-field gel electrophoresis patterns of XbaIdigested genomic DNA of Salmonella Enteritidis isolates. Lane 1, Salmonela Enteritidis clinical isolates recovered at Gulhane Military Medical Academy and confirmed at Refik Saydam National Hygiene Center; Lane 2, food isolate; Lane 3 , blood isolate; Lanes 4 to 11, stool isolates; Lane 12, repeated food isolate. Molecular size (SM) is given in kilobases.

\section{Microbiological analysis}

In this study stool samples collected from 445 (18\% of the 2,469 cases) hospitalized soldiers having gastroenteritis were analyzed for Salmonella spp. Of them, 276 (11.1\% of the 2,469 cases) had laboratory confirmed $S$. Enteritidis. $S$. Enteritidis strain was isolated from one of hospitalized patient's blood culture samples. All employees working in the kitchen were asymptomatic and their stool sample cultures were negative for Salmonella spp. No employee declared having had diarrhea or other gastrointestinal symptoms during the previous three months. All food and environmental samples from the kitchen submitted for microbiological analyses were negative for Salmonella spp. except for mashed potatoes with egg. All stool isolates, one blood isolate and one food isolate had the same antibiotic susceptibility pattern and were susceptible to all drugs tested which were ampicillin $(\leq 4 \mu \mathrm{g} / \mathrm{mL})$, amoxicillin/clavulanate $(\leq 4 / 2$ $\mu \mathrm{g} / \mathrm{mL})$, cefotaxime $(\leq 4 \mu \mathrm{g} / \mathrm{mL})$, chloramphenicol $(\leq 4 \mu \mathrm{g}$ ) $\mathrm{mL}$ ), trimethoprim/sulfamethoxazole (SXT) $(\leq 0.5 / 95 \mu \mathrm{g} /$ $\mathrm{mL})$, tetracycline $(\leq 2 \mu \mathrm{g} / \mathrm{mL})$, and ciprofloxacin $(\leq 0.5 \mu \mathrm{g}$ / $\mathrm{mL})$.

\section{PFGE and rep-PCR using DiversiLab}

The PFGE patterns after digestion with XbaI, and RepPCR using the DiversiLab system were performed for randomly eight selected stool isolates, one blood and one food isolate. All isolates had the same PFGE pattern (Fig. 1). The DiversiLab system showed that all isolates, were genotypically indistinguishable having $>95 \%$ similarity (Fig. 2). The PFGE and the DiversiLab system interpretations were concordant for $S$. Enteritidis food-borne outbreak isolates. The DiversiLab system allowed a complete microbial typing analysis in approximately 4 hours compared to 3 days for PFGE in our study. 


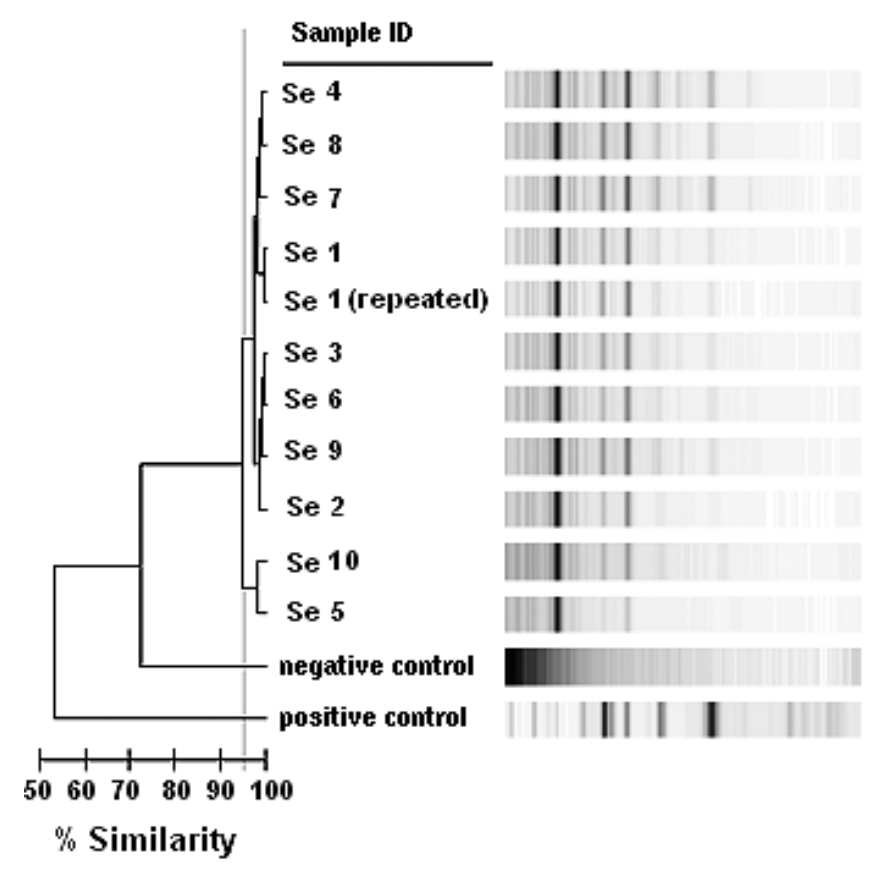

Figure 2. The DiversiLabb systems result (dendrogran and gel-like image) for Salmonella Enteritidis. Se 1, food iso. late; Se 2, blood isolate; Se 3 to 10, stool isolates.

\section{Discussion}

To our knowledge, this is the first report of $S$. Enteritidis food-borne outbreak from Turkey investigating the source and route by conventional and molecular microbiological methods.

Salmonella cause an estimated millions of illnesses and hundreds of deaths annually in many countries (21). S. Enteritidis has been one of the most frequently isolated Salmonella associated with food-borne outbreak (22). In the United States, a total of 121 Salmonella outbreaks occurred in 2006, causing greater than 3,300 illnesses reported to the CDC Foodborne Outbreak Reporting System. The most common outbreak serotypes were $S$. Enteriditis and $S$. Typhimurium (23). A total of 6,860 food-borne outbreaks were reported in European Union in 2004. Salmonella was the most frequently reported cause for these outbreaks $(73.9 \%$ of the reported outbreaks). $S$. Enteritidis were the predominant serotype associated with outbreaks where the serotype was reported (24). Since surveillance studies about foodborne outbreaks are newly established in Turkey, there is not sufficient documentation on Salmonella outbreaks. Tansel et al, described a $S$. Enteritidis food-borne outbreak which occurred in a military battalion located at the north-east of Turkey. The causative strain affected 60 soldiers in the battalion, 16 of whom hospitalized in the first month of the study (25). In the present study, 2,469 soldiers (445 of them hospitalized) were affected from the outbreak caused by $S$. Enteritidis. We should be aware that $S$. Enteritidis is the most common Salmonella serotype and may cause large food-borne outbreak in Turkey.
Eggs are especially known to be a source of $S$. Enteritidis infections in humans. S. Enteritidis contaminate eggs both external by during passage through the hen cloaca and internal by penetration through the eggshell via microscopic cracks. In the United States, either primarily egg-based or that which contained egg ingredients has been described as the source of $75 \%$ of food-borne outbreak with a confirmed food vehicles from 1985 to 2003. Although it is not possible to determine the source of infection for most individual case of salmonellosis, the Centers for Disease Control and Prevention has estimated that 50,000-110,000 cases of $S$. Enteritidis infection are attributed to eggs each year in the United States (26). Also in European Union, eggs, bakery products and meat products were the most important sources, and in approximately $50 \%$ of the food-borne outbreaks exposure took place in private homes or restaurants (24). In Turkey, Tansel et al reported a food-borne outbreak causing $S$. Enteritidis. An egg-containing omelet was considered to be the source of outbreak based on the epidemiological proof (25). In our study, undercooked mashed potatoes with egg were described as the source of the outbreak. We suggest that food items containing eggs should be cooked thoroughly to avoid of food-borne outbreak caused by $S$. Enteritidis which is the most common egg-associated pathogen.

Since its emergence, the description of outbreak in shorttime, tracing transmission routes and defining relationship between humans and food items have demonstrated to be very important (10). Because of the genetic homogeneity of $S$. Enteritidis isolates, a single method cannot be reliable for epidemiological analysis of unrelated and related strains of $S$. Enteritidis (2, 27-29). Therefore, reliable, sensitive and specific molecular epidemiologic methods are needed for the prevention and control of outbreaks caused by $S$. Enteritidis. Amplified fragment length polymorphism, random amplified polymorphic DNA, single-enzyme ribotyping analysis has been used subtyping for surveillance and investigation of food-borne outbreaks attributable to $S$. Enteritidis $(2,10,30)$. Each of these methods has limited discriminatory power, poor reproducibility and, in addition difficult to standardize and interlaboratory comparison (10). It was reported that for multi-locus variable-number tandem repeat analysis its is a useful tool for detection and investigation of outbreak caused by $S$. Enteritidis $(2,10,17,27)$. PFGE method has been proven to be useful in determining the relatedness of $S$. Enteritidis isolates and investigation of outbreak as currently gold standard (31-34). However, PFGE is mainly a complex and time-consuming procedure and there is reduced comparability of results between different laboratories. It also exhibits limited discriminatory power for $S$. Enteritidis $(10,17)$. The Rep-PCR using the DiversiLab system is a genotyping technique that provides good resolution between multiple strains within a single species (35). Recent studies on Rep-PCR using DiversiLab system have been published for bacteria such as Staphyloccocus aureus (36), Acinetobacter baumannii (13), vancomycin-resistant entero- 
cocci (20), Streptococcus pneumoniae (37), and Escherichia coli (38). In our study, the PFGE and the DiversiLab system interpretations were concordant for food-borne outbreak isolates. The DiversiLab system completed analysis of 10 samples in approximately 4 hours, compared to 3 days for PFGE. The DiversiLab system was shown to be technically simpler and more easily learned than PFGE. In addition the DiversiLab system, provides an option to digitalize gel images that can be archived in the web-based software, providing for easier comparison of samples between international laboratories.

We suggest that eggs containing food items should not be consumed raw or undercooked. Molecular epidemiologic methods can significantly contribute to a more reliable interpretation of food-borne outbreak caused by $S$. Enteritidis. The DiversiLab system may be a reasonable alternative to PFGE for surveillance and outbreak studies for $S$. Enteritidis, since it provides a simple, rapid and highly specific screening method that archives all gel image data.

\section{Acknowledgement}

We thank Ridvan Zeybek for transporting some samples from Isparta to our lab and Ali Bora Bas for providing free DiversiLab system kits.

\section{References}

1. D’Ortenzio E, Weill FX, Ragonneau S, Lebon JA, Renault P, Pierre V. First report of a Salmonella enterica serovar Weltevreden outbreak on Reunion Island, France, August 2007. Euro Surveill 13: 18949, 2008.

2. Pang JC, Chiu TH, Chiou CS, et al. Pulsed-field gel electrophoresis, plasmid profiles and phage types for the human isolates of Salmonella enterica serovar Enteritidis obtained over 13 years in Taiwan. J Appl Microbiol 99: 1472-1483, 2005.

3. Patrick ME, Adcock PM, Gomez TM, et al. Salmonella enteritidis infections, United States, 1985-1999. Emerg Infect Dis 10: 1-7, 2004.

4. de Jong B, Ekdahl K. The comparative burden of salmonellosis in the European Union member states, associated and candidate countries. BMC Public Health 6: 4, 2006.

5. Erdem B, Ercis S, Hascelik G, Gur D, Aysev AD. Antimicrobial resistance of Salmonella enterica group C strains isolated from humans in Turkey, 2000-2002. Int J Antimicrob Agents 26: 33-37, 2005.

6. Olson AB, Andrysiak AK, Tracz DM, et al. Limited genetic diversity in Salmonella enterica serovar Enteritidis PT13. BMC Microbiol 7: 87, 2007.

7. Matsuoka DM, Costa SF, Mangini C, et al. A nosocomial outbreak of Salmonella enteritidis associated with lyophilized enteral nutrition. J Hosp Infect 58: 122-127, 2004.

8. Thiagarajan D, Saeed AM, Asem EK. Mechanism of transovarian transmission of Salmonella enteritidis in laying hens. Poult Sci 73: 89-98, 1994.

9. Liebana E, Garcia-Migura L, Breslin MF, Davies RH, Woodward MJ. Diversity of strains of Salmonella enterica serotype enteritidis from English poultry farms assessed by multiple genetic fingerprinting. J Clin Microbiol 3: 154-161, 2001.

10. Boxrud D, Pederson-Gulrud K, Wotton J, et al. Comparison of multiple-locus variable-number tandem repeat analysis, pulsedfield gel electrophoresis, and phage typing for subtype analysis of Salmonella enterica serotype Enteritidis. J Clin Microbiol 45: 536543, 2007.

11. Olive DM, Bean P. Principles and applications of methods for DNA-based typing of microbial organisms. J Clin Microbiol 37: 1661-1669, 1999.

12. Versalovic J, Schneider M, de Brujin FJ, Lupski JR. Genomic fingerprinting of bacteria using repetitive sequence based PCR (repPCR). Meth Mol Cell Biol 5: 25-40, 1994.

13. Fontana $\mathrm{C}$, Favaro $\mathrm{M}$, Minelli $\mathrm{S}$, et al. Acinetobacter baumannii in intensive care unit: a novel system to study clonal relationship among the isolates. BMC Infect Dis 8: 79, 2008.

14. Wise MG, Siragusa GR, Plumblee J, Healy M, Cray PJ, Seal BS Predicting Salmonella enterica serotypes by repetitive sequencebased PCR. J Microbiol Methods 76: 19-24, 2009.
15. Spiliopoulou I, Zografou S, Goula A, Dimitracopoulos G, Christofidou M. Molecular epidemiology and antibiotic resistance patterns of Salmonella enterica from southwestern Greece. Chemotherapy 53: 392-396, 2007.

16. Joseph CA, Palmer SR. Outbreaks of Salmonella infection in hospitals in England and Wales 1978-87. BMJ 298: 1161-1164, 1989.

17. Beranek A, Mikula C, Rabold $P$, et al. Multiple-locus variablenumber tandem repeat analysis for subtyping of Salmonella enterica subsp enterica serovar Enteritidis. Int J Med Microbiol 299: 43-51, 2009.

18. Dice LR. Measures of the amount of ecological association between species. Ecology 26: 297-302, 1945.

19. Kilic A, Demiray T, Saracli MA, et al. Bacteriological characterization of vancomycin-resistant enterococci in a pediatric hospital in Turkey. Ann Microbiol 54: 543-551, 2004.

20. Pounder JI, Shutt CK, Schaecher BJ, Woods GL. Clinical evaluation of repetitive sequence-based polymerase chain reaction using the Diversi-Lab System for strain typing of vancomycin-resistant enterococci. Diagn Microbiol Infect Dis 54: 183-187, 2006.

21. Centers for Disease Control and Prevention (CDC). Multistate outbreaks of Salmonella infections associated with raw tomatoes eaten in restaurants--United States, 2005-2006. MMWR Morb Mortal Wkly Rep 56: 909-911, 2007.

22. Zheng J, Keys CE, Zhao S, Meng J, Brown EW. Enhanced subtyping scheme for Salmonella enteritidis. Emerg Infect Dis 13: 1932-1935, 2007.

23. Centers for Disease Control and Prevention (CDC). Annual Listing of Foodborne Disease Outbreaks, United States. http:// wwwcdcgov/foodborneoutbreaks/documents/2006_line_list/2006_ line_listpdf [Accessed 15 December 2008].

24. European Food Safety Authority (EFSA). Trends and sources of zoonoses, zoonotic agents and antimicrobial resistance in the European Union in 2004. http://wwwefsaeuropaeu/cs/BlobServer/ Report/zoonoses2004-levels1-2-part11pdf?ssbinary=true [Accessed 15 December 2008].

25. Tansel O, Ekuklu G, Otkun M, Otkun MT, Akata F, Tuğrul A. Food-borne outbreak caused by Salmonella enteritidis. Yonsei Med J 44: 198-202, M 2003.

26. Braden CR. Salmonella enterica serotype Enteritidis and eggs: a national epidemic in the United States. Clin Infect Dis 43: 512517, 2006.

27. Cho S, Whittam TS, Boxrud DJ, Bartkus JM, Saeed AM. Allele distribution and genetic diversity of VNTR loci in Salmonella enterica serotype Enteritidis isolates from different sources. BMC Microbiol 8: 146, 2008.

28. Thong KL, Ngeow YF, Altwegg M, Navaratnam P, Pang T. Molecular analysis of Salmonella enteritidis by pulsed-field gel electrophoresis and ribotyping. J Clin Microbiol 33: 1070-1074, 1995. 
29. Landeras E, González-Hevia MA, Alzugaray R, Mendoza MC Epidemiological differentiation of pathogenic strains of Salmonella enteritidis by ribotyping. J Clin Microbiol 34: 2294-2296, 1996.

30. Romani C, Nicoletti $P$, Buonomini MI, Nastasi A, Mammina C Reinterpreting a community outbreak of Salmonella enterica serotype Enteritidis in the light of molecular typing. BMC Public Health 7: 237, 2007.

31. Tassios PT, Markogiannakis A, Vatopoulos AC, et al. Molecular epidemiology of antibiotic resistance of Salmonella enteritidis during a 7-year period in Greece. J Clin Microbiol 35: 1316-1321, 1997.

32. Biendo M, Laurans G, Thomas D, et al. Regional dissemination of Salmonella enterica serovar Enteritidis is season dependent. Clin Microbiol Infect 9: 360-369, 2003.

33. Markogiannakis A, Tassios PT, Lambiri M, et al. Multiple clones within multidrug-resistant Salmonella enterica serotype Typhimurium phage type DT104 The Greek Nontyphoidal Salmonella Study Group. J Clin Microbiol 38: 1269-1271, 2000.
34. Biendo M, Laurans G, Thomas D, et al. Molecular characterisation and mechanisms of resistance of multidrug-resistant human Salmonella enterica serovar Typhimurium isolated in Amiens (France). Int J Antimicrob Agents 26: 219-229, 2005.

35. Cleland D, Krader P, Emerson D. Use of the DiversiLab repetitive sequence-based PCR system for genotyping and identification of Archaea. J Microbiol Methods 73: 172-178, 2008.

36. Ross TL, Merz WG, Farkosh M, Carroll KC. Comparison of an automated repetitive sequence-based PCR microbial typing system to pulsed-field gel electrophoresis for analysis of outbreaks of methicillin-resistant Staphylococcus aureus. J Clin Microbiol 43: 5642-5647, 2005.

37. Harrington SM, Stock F, Kominski AL, et al. Genotypic analysis of invasive Streptococcus pneumoniae from Mali, Africa, by semiautomated repetitive-element PCR and pulsed-field gel electrophoresis. J Clin Microbiol 45: 707-714, 2007.

38. Vogel JR, Stoeckel DM, Lamendella R, et al. Identifying fecal sources in a selected catchment reach using multiple sourcetracking tools. J Environ Qual 36: 718-729, 2007.

(C) 2010 The Japanese Society of Internal Medicine http://www.naika.or.jp/imindex.html 